\title{
Resonance Frequency Prediction of Aperture Coupled Microstrip Antenna Based on Artificial Neural Network
}

\author{
Mariem Abdi ${ }^{1, *}$ Taoufik Aguili ${ }^{2}$ \\ 1,2 Syscom Laboratory, National Engineering School of Tunis, Tunis El Manar university BP 37, le Belvédère, \\ Tunisia \\ *Corresponding author. Email: mariemahmedabdi@gmail.com
}

\begin{abstract}
Communication in the millimeter frequency band becomes a requirement with the launch of $5 \mathrm{G}$ hence the importance of the design of integrated antennas. The aperture-coupled microstrip patch antenna is a suitable choice for 5G application especially since it presents less losses compared to others microstrip antennas. This antenna must be adapted to the resonant frequency by operating in the frequency band allocated for automotive radar applications ranging from $77 \mathrm{GHz}$ to $81 \mathrm{GHz}$. This article presents the prediction of the resonant frequency of an aperture coupled fed microstrip patch antenna in function of its physical input parameters by proposing two models. The first model is the 3D HighFrequency Electromagnetic Simulation Software model. The second model is the Artificial Neural Network learning model and the Levenberg-Marquardt which is adopted as a learning algorithm. The results revealed that the Artificial Neural Network learning model works at least ten thousand times faster than the High Frequency Electromagnetic Simulation Software model. It was further concluded that it can be used as a preliminary research tool to optimize design models for the aperture coupled microstrip patch antenna due to its speed of operation due to its overwhelming operating speed.
\end{abstract}

Keywords: Aperture, Coupled, Microstrip, Patch, Antenna, Radar, Software, Model, Neural, Network, Learning, Levenberg, Marquardt, Resonant, Frequency.

\section{INTRODUCTION}

To satisfy the $5 \mathrm{G}$ requirement, it is crucial that the antenna technology is miniature and low cost. Indeed, the microstrip antenna seems to be the most suitable technology for $\mathrm{mm}$-wave $5 \mathrm{G}$ applications. The aperturecoupled fed microstrip patch antenna (ACMPA) offers many advantages as determined in various research works [1] [2] [3]. The major advantage of this non-direct feed technique is that it minimizes the effect of frequency band losses compared to antennas excited by direct feed. The adaptation of the antenna is a crucial parameter so that the antenna can resonate at the requested resonant frequency. This operation was largely effectuated with the High Frequency Software Simulator (HFSS). As a result of the large computational load generated by the simulation programs used to obtain the suitable antenna parameters, new computer-aided methods have been developed such as the machine learning algorithms known by the artificial neural network. In fact, in recent years, the artificial neural network learning models (ANN) have become popular because of their rapid learning abilities, accessible adaptability to diverse problems, performance with little data, and ability to generalize [4]. The Levenberg-Marquardt (LM-ANN) is applied for prediction of the output parameter which is the resonant frequency of ACMPA. The frequency is dependent on the geometric parameters of the patch's width and length, the excitation line's length, the 
aperture's length and width. LM-ANN prediction performance and execution speed are evaluated with HFSS commercial software.

The first step is to present the methodology problem statement. In Section III, the description of the antenna is presented. In Section IV, the LM-ANN artificial neural network modelling is illustrated. Section V presents the numerical results are given and analysed. The last section contains a conclusion.

\section{METHODOLOGY STATEMENT}

PROBLEM

Microstrip antennas are considered among the most widely used antennas in millimeter band applications for their variable size, low cost, easy modeling, and wide range of resonant frequencies. In this paper, a rectangular patch antenna with coupling feed was chosen for its wide acceptability of its transmission line pattern analysis.

In fact, this model is based on relatively complex mathematical formulas to find the physical parameters (length and width) according to certain design parameters (resonance frequency and height of the substrate and its dielectric constant).

Certainly, the calculations performed through the transmission line model are heavy and time consuming, and this is where the role of Artificial Neural Networks (ANNs) comes in [5] [6] [7]. In this context, the prediction of the resonant frequency is carried out through the two models previously mentioned in the introduction. At the resonant frequency, the reflection coefficient $S_{11} \leq-20 \mathrm{~dB}$. The automotive radar application requires the antenna to be adapted in the frequency band operating from 77 Gigahertz to 81 Gigahertz.

\section{ANTENNA DESCRIPTION}

In 1985, D.M. Pozar has introduced the aperture coupling technique [8]. The Aperture Coupled Fed Microstrip Patch Antenna (ACMPA) is formed by two substrates separated through a ground plane pierced with aperture. As shown in Figure 1, a radiating patch is positioned in the top of the substrate while the exciter line is positioned in the lower substrate.

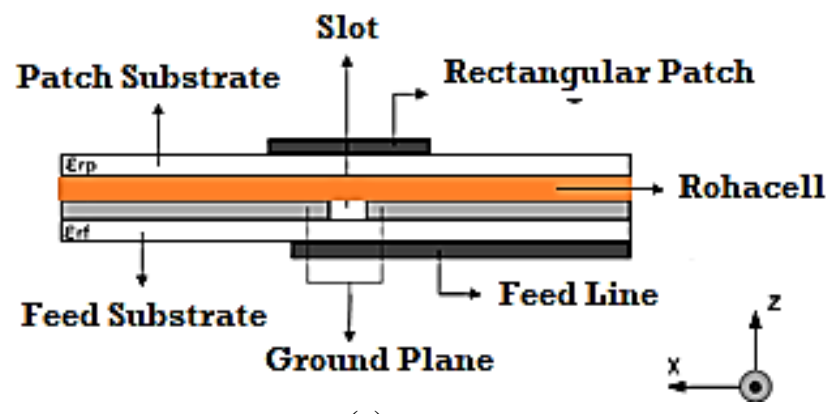

(a)

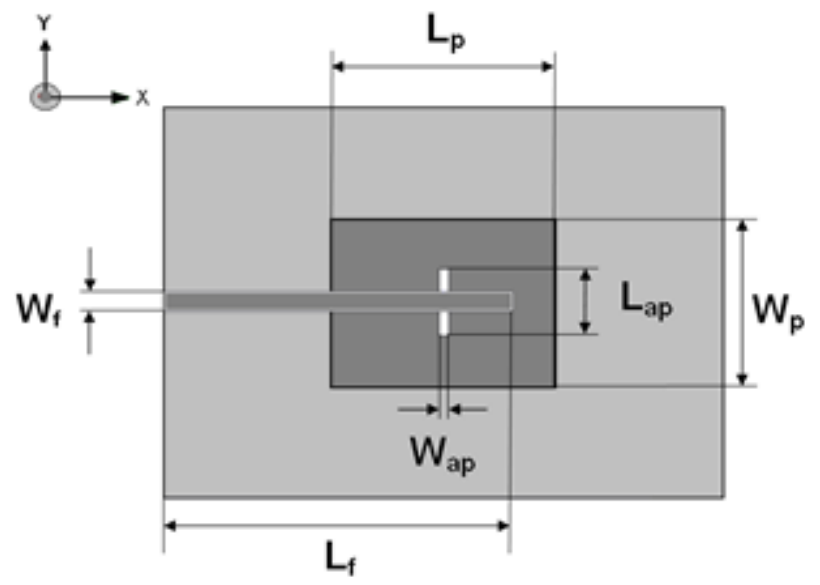

(b)

Figure 1 Aperture Coupled Microstrip Patch Antenna (a) Side view (b) Bottom view.

The antenna parameters of the antenna are presented by:

- $\quad$ Radiating Patch dimensions

- $\quad L_{p}$ : length of the patch

- $\quad W_{p}$ : width of the patch

- Aperture dimensions:

$$
\text { - } \quad L_{a p} \text { : aperture length }
$$

- Feed dimensions:

- $\quad$ Lf: $_{\mathrm{f}}$ excitation line length

- $\quad W_{f}$ : excitation line width

The electromagnetic fields are grouped tightly under the slit to be "directed" from the exciter line to the patch antenna without any physical connection as mentioned in Figure 2. Coupling becomes maximum when the patch is centered on the slot [9]. The dimensions and shape of the slot influence the coupling mechanism as well as the dimensions parameters of the patch and the excitation transmission line length.

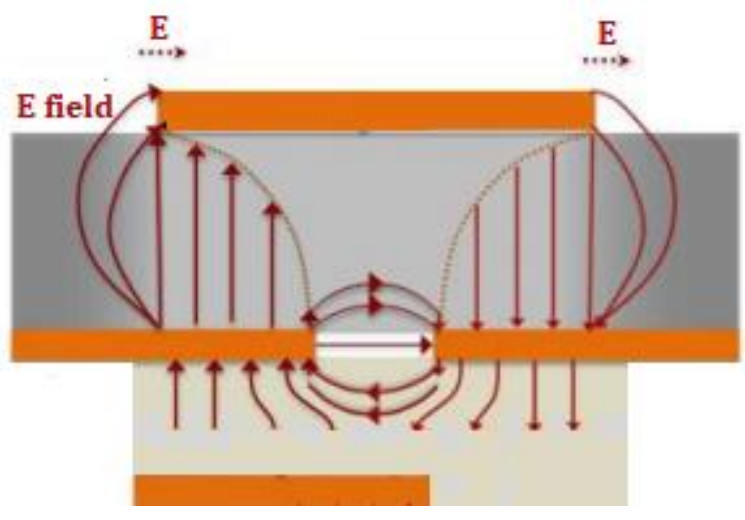

Figure 2 Principle of the Aperture Coupled Fed Microstrip Patch Antenna. 


\section{ARTIFICIAL NEURAL NETWORK}

Neural networks are abstract constructs simulating the activity of a simplified biological neural network. They are used in machine learning to build a model from existing data in order to make predictions on new data either using regression, in the continuous case, or classification, in the discrete case. A more complete introduction to machine learning and its cousin data mining is presented in [10] [11]. Machine learning is about adjusting the parameters of a "box" that takes data as input and outputs a result. This is the classic metaphor for describing a function. In statistics, the box is often simple and can use mathematical analysis to characterize the optimal parameters. For example, classical regression uses a "box" made up of a linear combination of relevant elementary functions and the fit is simply to optimize the coefficients of the linear combination (see Figure 3 ).

The modern context of data mining has motivated the development of more complex "boxes", impossible to optimize analytically. Neural networks are an example of such complex models which have proved their worth in several fields such as fraud detection or the recognition of handwritten characters. The neurons of the input layer $i$ distribute the signals' input $X i$ to the hidden layer's neurons. The hidden layer's neuron $j$ combines the signals' input $X_{i}$ by weighting them through the connections' power $W_{j i}$ then computes the output $y_{i}$. The neurons' output in the output layer is calculated in the same way. The activation function applied at the input of the neuron can be expressed as below [12]:

$\mathrm{y}_{\mathrm{i}}=\mathrm{a}\left(\sum \mathrm{w}_{\mathrm{ji}} \mathrm{x}_{\mathrm{i}}+\mathrm{b}_{\mathrm{i}}\right)$

The geometric parameters of ACMPA $\left(L_{p}, W_{p}, L_{a p}\right.$, $\left.W_{a p}, L_{f}\right)$ were taken into account. The dielectric constant and the height of the dielectric material used were conserved constant. The Rogers RT / duroid 5880 тм dielectric material was used to model the two dielectric layers having a dielectric permittivity worth 2.2 and a loss tangent worth 0.0009 .

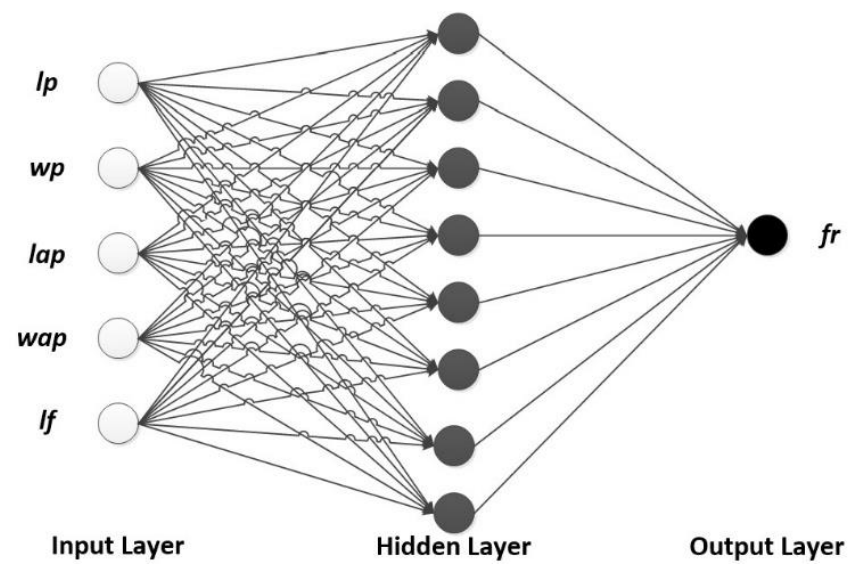

Figure 3 MPL basis structure of proposed neural structure.
Initially, a neural network with randomly initialized weights gives poor results. This is why there is a learning step, that is, adjusting the weights of the network so that they can give better predictions. One way to look at the problem is to try to minimize the model's prediction error. This formulation transforms the learning problem into an optimization problem for which several algorithms exist. Optimization of the error function is accomplished by adjusting the parameters of the function, that is, the weights and biases of the neural network.

Percentage error formula is expressed as a generalization performance metric:

Error(percentage $)=\left|\frac{\mathrm{fr}(\mathrm{HFSS})-\mathrm{fr}(\mathrm{LM}-\mathrm{ANN})}{\mathrm{fr}(\mathrm{HFSS})}\right| \times 100$

Reflexion coefficient must be parted into three sets:

- The raining set which is used to complete LM-ANN weights.

- The validation set which is used to validate the LMANN performance and decide when to break the training process.

- The test set, which is used to determine the performance capabilities of the LM-ANN model.

\section{SIMULATED RESULTS AND DISCUSSION}

\subsection{HFSS Dataset Generation}

Based on the HFSS software, the data set for this study consists of 500 samples. Any sample in the dataset combines five ACMPA input parameters $\left(L_{p}, W_{p}, L_{a p}\right.$, $\left.W_{a p}, L_{f}\right)$ and one output parameter which is the resonant frequency (fr) (see Figure 4).

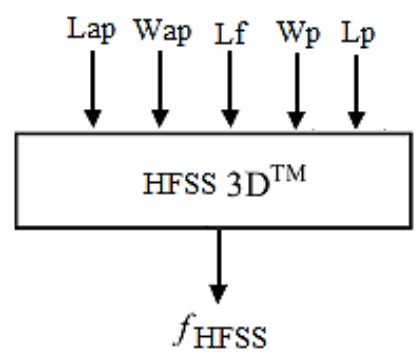

Figure 4 HFSS Proposed Simulation Model.

Some output of resonant frequency values in terms of the ACMPA input parameters used in HFSS dataset are listed in Table 1. 
Table 1. HFSS software's dataset

\begin{tabular}{c|c|c|c|c|c|c}
\multirow{2}{*}{\begin{tabular}{c} 
Model Number \\
\cline { 2 - 7 }
\end{tabular}} & \multicolumn{7}{|c|}{$\mathrm{L}_{\mathrm{p}}$} & $\mathrm{W}_{\mathrm{p}}$ & $\mathrm{L}_{\mathrm{ap}}$ & $\mathrm{W}_{\mathrm{ap}}$ & \multicolumn{2}{c}{$\begin{array}{c}\text { Output Parameter } \\
\text { (GHz) }\end{array}$} \\
\cline { 2 - 7 } & 1.349 & 1.8 & 1.2 & 0.234 & 2 & 79 \\
\hline 1 & 1.254 & 1.72 & 1.95 & 0.238 & 1.94 & 79.20 \\
\hline 3 & 1.3 .21 & 1.74 & 1.88 & 0.241 & 1.85 & 79.82 \\
\hline 4 & 1.238 & 1.68 & 1.82 & 0.252 & 1.81 & 80.48 \\
\hline 5 & 1.220 & 2.04 & 1.99 & 0.97 & 2.01 & 81.02 \\
\hline
\end{tabular}

\subsection{LM-ANN application}

To visualize the behavior of the antenna at resonance, a study on the coefficient of reflection parameter versus frequency was developed on a frequency band ranging from $60 \mathrm{GHz}$ to $100 \mathrm{GHz}$ (Figure 5). At the resonance frequency, the antenna is adapted, i.e., the reflection coefficient $S_{11} \leq-20 \mathrm{~dB}$. The artificial neural network training parameters are illustrated in Table 2.

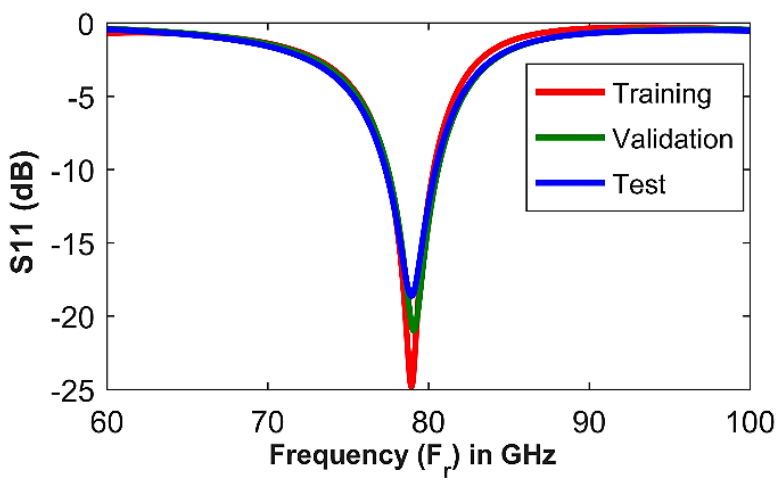

Figure 5 The coefficient reflexion parameter versus frequency. The parameters which chosen to simulate the suggested $S_{11}: L_{p}=1.349, W_{p}=1.8, L_{a p}=1.2$, $W_{a p}=0.234, L f=2$.

Table 2. Artificial neural network training parameters

\begin{tabular}{c|c|}
\hline Model Number & $\begin{array}{c}\text { Output Parameter } \\
\text { (GHz) }\end{array}$ \\
\hline Number of input neurons & 5 \\
\hline Hidden layers' number & 8 \\
\hline Output neurons' number & 1 \\
\hline Learning Algorithm & $10^{-2}$ \\
\hline Learning rate & $95 \times 10^{-2}$ \\
\hline Momentum & $1 e^{-1}$ \\
\hline Mean Squared Error goal & 9 \\
\hline Maximum validation failures & $1 e^{-5}$ \\
\hline Minimum performance gradient & $10^{-3}$ \\
\hline Lnitial control parameter for the LM-ANN for training the neural network & $10^{-1}$ \\
\hline Decrease factor & 10 \\
\hline Increase factor & $1 e^{-10}$ \\
\hline Maximum control parameter for the LM-ANN for training the neural network & 27 \\
\hline Epochs among displays & false \\
\hline Command-line output Generation & true \\
\hline inf \\
\hline Training GUl Shown & 450 \\
\hline Eaximum running time per seconds & $8 \times 10^{-1}$ \\
\hline Regularization parameter & tansig \\
\hline Transfer function (hidden layer) & purelin
\end{tabular}

The study was held on a computer equipped with an Intel Pentium i7-7500U, 2.7 Gigahertz processor, 8 Gigabit of memory and the Windows 10 as an operating system. Table 3 includes input parameters which have been added to Table 1 and presents the results obtained from the ANN and HFSS model. The output values observed for the resonant frequency are consistent with an acceptable error percentage (see Figure 6).

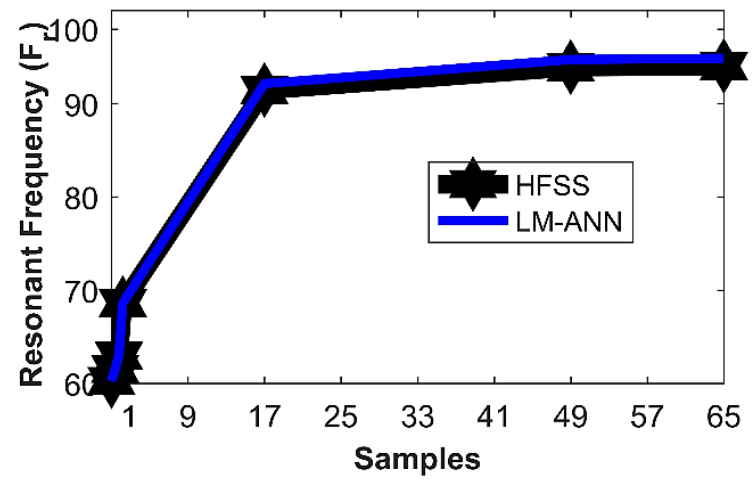

Figure 6 Test result comparison of the proposed LMANN model over the HFSS model. 
Table 3. Results and percentage error rates of the two models: HFSS and LM-ANN

\begin{tabular}{c|c|c|c|c|c|c|c|c|} 
& \multicolumn{5}{|c|}{} & \multicolumn{2}{c|}{$\begin{array}{c}\text { Output Parameter } \\
(\mathrm{GHz})\end{array}$} \\
\cline { 2 - 9 } & $\mathrm{L}_{p}$ & $\mathrm{~W}_{\mathrm{p}}$ & $\mathrm{L}_{\mathrm{ap}}$ & $\mathrm{W}_{\mathrm{ap}}$ & $\mathrm{Lf}_{\mathrm{f}}$ & HFSS & ANN & \\
\hline 1 & 1.349 & 1.8 & 1.2 & 0.234 & 2 & 79 & 79.1 & 0.01 \\
\hline 2 & 1.254 & 1.72 & 1.95 & 0.238 & 1.94 & 79.20 & 79.18 & 0.22 \\
\cline { 2 - 9 } & 1.3 .21 & 1.74 & 1.88 & 0.241 & 1.85 & 79.82 & 79.85 & 0.03 \\
\hline 4 & 1.238 & 1.68 & 1.82 & 0.252 & 1.81 & 80.48 & 80.36 & 0.14 \\
\hline 5 & 1.220 & 2.04 & 1.99 & 0.97 & 2.01 & 81.02 & 81.11 & 0.11 \\
\hline
\end{tabular}

Table 4. Processing time evaluation of the LM-ANN and the HFSS simulation models

\begin{tabular}{c|c|c}
\hline & Average Time & Standard Deviation \\
\hline HFSS & 40.16 & 25.85 \\
\hline LM-ANN & 0.0035 & 0.001 \\
\hline
\end{tabular}

The comparison of the HFSS and the proposed simulation model according to the running speed is listed in Table 4 above. The prediction model based on LMANN is much more powerful as function of computational time and speed than the HFSS simulation software. This indicates that the proposed ANN model is more promising for the resonant frequency prediction of the ACMPA antenna.

\section{CONCLUSION}

In this paper, the LM-ANN output parameters which are the resonance frequency values of the Aperture Coupled Fed Microstrip Patch Antenna were predicted while comparing to those obtained with the HFSS simulation. The assessment of processing time of LMANN and HFSS simulation models prove that the LMANN simulation is much more powerful in terms of simulation time and speed than the HFSS simulation software.

LM-ANN model predicts a single output parameter. It is planned in future research to handle a study that introduces more antenna parameters namely the gain, the bandwidth, the VSWR, the radiation pattern ...

This prediction will have an impact in terms of time and cost when modeling and designing the antenna.

\section{REFERENCES}

[1] H. Al-Saedi, J. Attari, W. M. Abdel Wahab, Raj Mittra and S. Safavi-Naeini, Single-feed Dual-band Aperture-coupled Antenna for 5G applications, in: 18th International Symposium on Antenna Technology and Applied Electromagnetics (ANTEM), 2018.2 DOI: 10.1109/ANTEM.2018.8572907

[2] P. Sullivan and D. Schaubert, Analysis of an aperture coupled microstrip antenna, in: IEEE Transactions on Antennas and Propagation, vol. 34, 1986, pp. 977 - 984. DOI: 10.4236/wet.2011.22013
[3] M. Abdi and T. Aguili, Design of a Compact Multilayered Aperture Coupled Microstrip Antenna for Automotive Range Radar Application, in: IEEE 19th Mediterranean Microwave Symposium (MMS), 2019.2 DOI: 10.1109/MMS48040.2019.9157286

[4] A. Rawata., Yadavb. R.N, and Shrivastavac. S.C, Neural network applications in smart antenna arrays: in: AEU - International Journal of Electronics and Communications, vol. 66, 2012, 903-912.

[5] D. M. Pozar, Considerations for millimeter wave printed antennas, in: IEEE Trans. on Antennas and Propagation, vol. 31, 1983, pp. 987-993. DOI: 10.1109/TAP.1983.1143124

[6] T. Bose1 and N. Gupta, Design of an Aperture Coupled Microstrip Antenna using Hybrid Neural Network, in: IET Microwaves Antennas \& Propagation, vol. 6, 2012, pp. 470-474. DOI:10.4236/wet.2010.12010

[7] T. Rao, R. Pandey and A. K. G. Kandu, Design Aperture Coupled Rectangular Microstrip Antenna at $28 \mathrm{GHz}$ and Bandwidth Calculation by using Artificial Neural Network for 5G Applications, in: Inetrnational journal of scientific progress and research (IJSPR), vol. 73, 2020, pp. 8489.

[8] M Kantipudi, S. Vemuri, S. Kashyap and Y. S. Kumar, Modeling of Microstrip Patch Antenna Using Artificial Neural Network Algorithms, in: Advanced Informatics for Computing Research, 2021.

[9] Y. Rao, H. Zhang and G. Sun , Shared Aperture Dual-band Waveguide Slot antenna, in: IEEE International Symposium on Antennas and Propagation and North American Radio Science 
Meeting, 2021, pp. 693-694.

DOI:10.1109/IEEECONF35879.2020.9330047

[10] M. F. Nakmouche, A.M.M.A. Allam, D. E. Fawzy, D. B. Lin , Low Profile Dual Band H-Slotted DGS Based Antenna Design Using ANN for K/Ku Band Applications, in: International Conference on Electrical and Electronics Engineering (ICEEE), 2021. DOI: 10.1109/ICEEE52452.2021.9415911

[11] H., Limam, S. and T.Aguili, Uniform and Concentric Circular Antenna Arrays Synthesis for Smart Antenna Systems Using Artificial Neural Network Algorithm, in: Progress in Electromagnetics Research B, vol. 67, 2016, pp. 91105. DOI:10.2528/PIERB16031508

[12] S.Rahul and N. Gupta, Electromagnetic Modeling of Microstrip Patch Antennas: A Neural Network Approach, in: International Conference for Convergence in Technology (I2CT), 2021. DOI: 10.1109/I2CT51068.2021.9418117 